\title{
Démonstration constructive de l'existence de polynômes de Bernstein-Sato pour plusieurs fonctions analytiques
}

\author{
Rouchdi Bahloul
}

\begin{abstract}
In 1987, Sabbah proved the existence of Bernstein-Sato polynomials associated with several analytic functions. The purpose of this paper is to give a more elementary and constructive proof of Sabbah's result based on the notion of the analytic Gröbner fan of a $\mathcal{D}$-module.
\end{abstract}

\section{Introduction et énoncé des résultats principaux}

Fixons $n \geqslant 1$ et $p \geqslant 1$ deux entiers et $v \in \mathbb{N}^{p} \backslash\{0\}$. Soient $x=\left(x_{1}, \ldots, x_{n}\right)$ et $s=\left(s_{1}, \ldots, s_{p}\right)$ deux systèmes de variables. On se donne $f_{1}, \ldots, f_{p} \in \mathbb{C}\{x\}=\mathbb{C}\left\{x_{1}, \ldots, x_{n}\right\}$. Notons $\mathcal{D}_{n}$ l'anneau des opérateurs différentiels à coefficients dans $\mathbb{C}\{x\}$. Pour $b(s) \in \mathbb{C}[s]=\mathbb{C}\left[s_{1}, \ldots, s_{p}\right]$, considérons l'identité suivante :

$$
b(s) f^{s} \in \mathcal{D}_{n}[s] f^{s+v},
$$

où $f^{s+v}=f_{1}^{s_{1}+v_{1}} \cdots f_{p}^{s_{p}+v_{p}}$. Un polynôme $b(s)$ vérifiant une telle identité est appelé polynôme de Bernstein-Sato (associé à $f=\left(f_{1}, \ldots, f_{p}\right)$ ). L'ensemble de ces polynômes forment un idéal appelé idéal de Bernstein-Sato (associé à $f$ ) et qu'on note $\mathcal{B}^{v}(f)$. Précisons que les cas les plus fréquemment étudiés sont ceux où l'on a $v=(1, \ldots, 1)$ ou bien $v=(0, \ldots, 0,1,0, \ldots, 0)$ où le 1 est en $j$-ième position pour un $j \in\{1, \ldots, p\}$.

Rappelons que c'est Bernstein [Ber72] qui, dans le cas $p=1$ et où $f$ est polynomiale, a montré que l'idéal $\mathcal{B}^{v}(f)$ est non nul (dans ce cas, il faut, dans $(\star)$, remplacer $\mathcal{D}_{n}$ par l'algèbre de Weyl $\mathbf{A}_{n}(\mathbb{C})$, i.e. l'algèbre des opérateurs différentiels à coefficients polynomiaux). Dans le cas où $f$ est analytique et toujours pour $p=1$, la non nullité de $\mathcal{B}^{v}(f)$ revient à Björk [Bjö73] avec des méthodes similaires à celles employées dans [Ber72]. Dans ce même cas, citons Kashiwara [Kas76] qui publia une autre preuve et démontra en plus que le générateur unitaire de l'idéal de Bernstein-Sato est à racines rationnelles. Pour $p \geqslant 2$, la preuve dans le cas polynomial est une généralisation facile de celle de Bernstein, que l'on peut trouver dans [Lic88]. Dans le cas analytique avec $p \geqslant 2$, la non nullité de $\mathcal{B}^{v}(f)$ a été démontrée par Sabbah [Sab87a, Sab87b]. Citons la contribution de Gyoja [Gyo93] qui a repris la preuve de Sabbah en montrant de plus que $\mathcal{B}^{v}(f)$ contient un élément rationnel non nul.

L'objet du présent article est une mise au point de la preuve donnée par Sabbah. Plus précisément, on peut décomposer la preuve de Sabbah en deux grandes étapes : la première utilise des arguments similaires à ceux employés par Kashiwara dans le cas $p=1$, la deuxième consiste essentiellement en un résultat de finitude qui permet par la suite de se ramener au résultat de la première étape. La seconde étape de la preuve de Sabbah s'appuie sur un éventail dit adapté

Received 16 July 2003, accepted in final form 21 January 2004, published online 1 December 2004.

2000 Mathematics Subject Classification 16S32, 13P10, 16W50, 16 W70.

Keywords: $V$-filtration, analytic Gröbner fan, Bernstein-Sato polynomial.

This journal is (C) Foundation Compositio Mathematica 2005. 


\section{R. BAHLOUL}

dont l'existence [Sab87a, théorème A.1.1] mériterait une mise au point technique supplémentaire. En effet, la démonstration du théorème A.1.1 de [Sab87a] est erronée (voir le commentaire qui suit le théorème S1). Aussi, nous proposons dans cet article un énoncé et une démonstation plus élémentaires et plus constructifs de la seconde étape, qui évitent la notion délicate d'éventail adapté.

Afin de motiver les résultats du présent article, nous rappelons, sans entrer dans tous les détails, la preuve de Sabbah. Dans ce rappel, nous mettrons en évidence le résultat de Sabbah pour lequel nous allons donner un énoncé et une preuve plus constructifs. Signalons que la plupart des notions ou notations introduites ci-dessous qui nous seront utiles dans la suite seront détaillées dans les sections suivantes.

On note $\mathcal{D}_{n+p}$ l'anneau des opérateurs différentiels à coefficients dans

$$
\mathbb{C}\{x, t\}=\mathbb{C}\left\{x_{1}, \ldots, x_{n}, t_{1}, \ldots, t_{p}\right\} .
$$

En suivant la méthode de Malgrange [Mal75], on fait agir $\mathcal{D}_{n+p}$ sur

$$
\mathbb{C}\{x\}\left[\frac{1}{f_{1} \cdots f_{p}}, s\right] f^{s} .
$$

On note $I$ l'idéal (à gauche ${ }^{1}$ ) annulateur de $f^{s}$ dans $\mathcal{D}_{n+p}$ et $M$ le quotient $M=\mathcal{D}_{n+p} / I$.

Pour chaque $j=1, \ldots, p$, notons $V_{j}\left(\mathcal{D}_{n+p}\right)$ la $V$-filtration de Kashiwara-Malgrange associée à la variable $t_{j}$ et notons $V=\left(V_{1}, \ldots, V_{p}\right)$ la (multi)filtration de $\mathcal{D}_{n+p}$ indexée par $\mathbb{Z}^{p}:$ pour $w \in \mathbb{Z}^{p}$,

$$
V_{w}\left(\mathcal{D}_{n+p}\right)=\bigcap_{j=1}^{p}\left\{V_{j}\right\}_{w_{j}}\left(\mathcal{D}_{n+p}\right) .
$$

Elle induit une filtration $V(M)$ sur $M$ où pour tout $w \in \mathbb{Z}^{p}, V_{w}(M)$ est l'image de $V_{w}\left(\mathcal{D}_{n+p}\right)$ par la projection $\mathcal{D}_{n+p} \rightarrow M=\mathcal{D}_{n+p} / I$.

Pour $j=1, \ldots, p$, identifions la filtration $V_{j}$ avec la forme linéaire sur $\mathbb{N}^{2 n+2 p}$ donnée par $V_{j}(\alpha, \mu, \beta, \nu)=\nu_{j}-\mu_{j}$ (où $\alpha, \beta \in \mathbb{N}^{n}, \mu, \nu \in \mathbb{N}^{p}$ et $\alpha, \mu, \beta, \nu$ correspondent respectivement à $\left.x, t, \partial_{x}, \partial_{t}\right)$. Notons $\mathcal{U}_{V}=\sum_{j=1}^{p} \mathbb{R}_{\geqslant 0} V_{j}$. On identifie $\mathcal{U}_{V}$ à $\left(\mathbb{R}_{\geqslant 0}\right)^{p}$. Chaque $L$ de $\mathcal{U}_{V} \cap \mathbb{N}^{p}$ (i.e. $L$ à coefficients entiers) donne lieu à une filtration naturelle $V^{L}$ de $\mathcal{D}_{n+p}$ et de $M$ indexée par $\mathbb{Z}$ donnée par

$$
V_{k}^{L}(M)=\sum_{\left\{w \in \mathbb{Z}^{p} ; L(w) \leqslant k\right\}} V_{w}(M),
$$

où $L(w)=l_{1} w_{1}+\cdots+l_{p} w_{p}$ si $L=\left(l_{1}, \ldots, l_{p}\right) \in \mathbb{N}^{p}$.

Voici maintenant les deux étapes de la preuve donnée par Sabbah.

\section{Etape 1.}

ThÉorème ([Sab87a, théorème 3.1.1], voir aussi [Gyo93, 2.9 et 2.10]). Pour tout $L \in \mathcal{U}_{V} \cap \mathbb{N}^{p}$, il existe un polynôme non nul $b \in \mathbb{C}[\lambda]$ d'une variable tel que pour tout $k \in \mathbb{Z}$, on ait

$$
b\left(L\left(-\partial_{t_{1}} t_{1}, \ldots,-\partial_{t_{p}} t_{p}\right)-k\right) V_{k}^{L}(M) \subset V_{k-1}^{L}(M) .
$$

La preuve de ce résultat utilise des arguments analogues à ceux employés par Kashiwara [Kas76] dans le cas $p=1$.

Notons $b_{L}$ le polynôme unitaire de plus bas degré satisfaisant l'identité précédente. La contribution de Gyoja [Gyo93] consiste dans le fait que $b_{L}$ est à racines dans $\mathbb{Q}<0$.

${ }^{1}$ Dans tout le texte, idéal signifie idéal à gauche. 
Existence De polynômes DE Bernstein-SAto

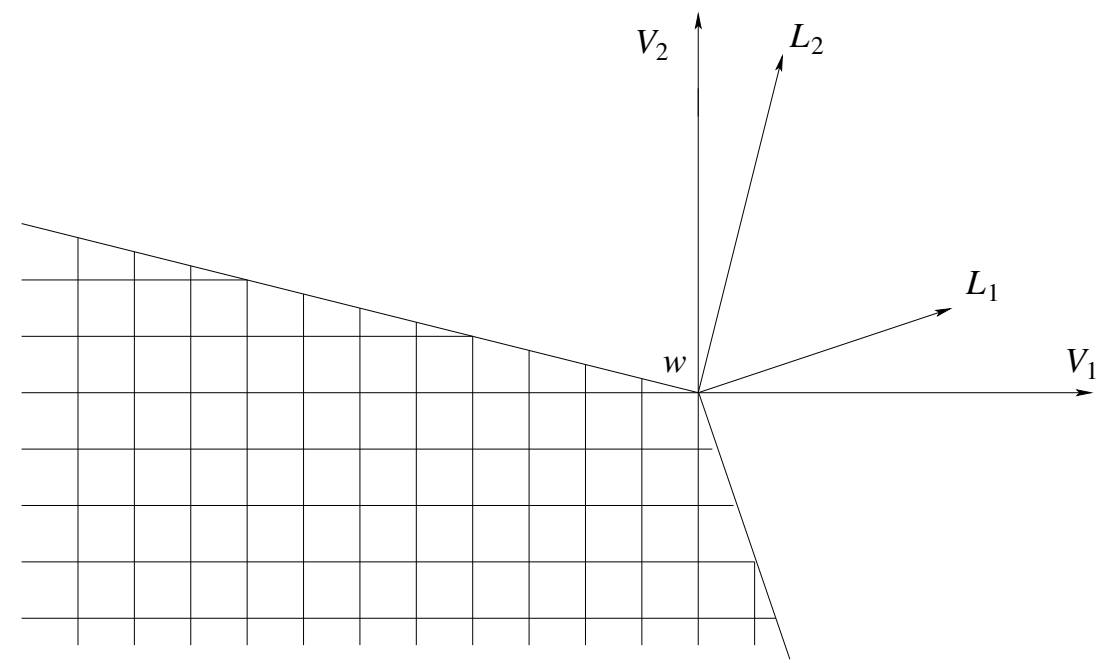

Figure 1. La filtration ${ }^{\sigma} V_{w}(M)$.

Etape 2. Nous allons introduire deux nouvelles filtrations sur $M$.

i) Soit $\sigma$ un cône convexe rationnel dans $\mathbb{R}_{\geqslant 0}^{p}$. On note $\mathcal{L}(\sigma)$ l'ensemble des éléments primitifs du 1-squelette de $\sigma$ (i.e. $L \in \mathcal{L}(\sigma)$ si et seulement si la droite engendrée par $L$ est dans le 1-squelette de $\sigma$ et les coefficients de $L$ sont des entiers sans facteurs communs). Pour tout $w \in \mathbb{Z}^{p}$, on note

$$
{ }^{\sigma} V_{w}(M)=\sum_{\left\{w^{\prime} \in \mathbb{Z}^{p} \mid \forall L \in \mathcal{L}(\sigma) L\left(w^{\prime}\right) \leqslant L(w)\right\}} V_{w^{\prime}}(M) .
$$

(Voir la figure 1 où $p=2$ et $\sigma$ est engendré par $L_{1}$ et $L_{2}: m \in{ }^{\sigma} V_{w}(M) \Longleftrightarrow m$ est représenté par un opérateur $P \in \mathcal{D}_{n+p}$ dont le diagramme de Newton est dans le quadrillage.)

Pour tout $w \in \mathbb{Z}^{p}$, nous avons facilement les inclusions suivantes :

$$
{ }^{\sigma} V_{w}(M) \subseteq \bigcap_{L \in \sigma} V_{L(w)}^{L}(M) \subseteq \bigcap_{L \in \mathcal{L}(\sigma)} V_{L(w)}^{L}(M),
$$

la première étant triviale et la seconde venant du fait que $\mathcal{L}(\sigma)$ est inclus dans l'adhérence de $\sigma$.

ThÉorème S1 [Sab87a, théorème A.1.1 et proposition 2.2.1]. Il existe un éventail $\Sigma$ (dit éventail adapté à $V(M)$ ) constitué de cônes polyédraux rationnels convexes tel que pour tout cône $\sigma \in \Sigma$ et pour tout $w \in \mathbb{Z}^{p}$, on ait

$$
{ }^{\sigma} V_{w}(M)=\bigcap_{L \in \mathcal{L}(\sigma)} V_{L(w)}^{L}(M)
$$

C'est ce théorème S1 dont nous allons donner un énoncé et une preuve plus élémentaires. Disons un mot de la preuve. Dans l'appendice de [Sab87a] en collaboration avec Castro Jiménez, Sabbah énonce l'existence d'un éventail $\Sigma$ dit adapté à la filtration $V(M)$ (théorème A.1.1) après quoi il montre dans la proposition 2.2.1 que pour tout cône d'un tel éventail, on a l'égalité précédente. La preuve du théorème A.1.1 sur l'existence d'un tel éventail comporte une erreur. En effet, elle s'appuie sur une division à paramètre qui conduirait à l'apparition de séries formelles dans les variables $\partial_{x_{i}}$ de dérivation.

ii) Soit $\bar{V}(M)$ la filtration indexée par $\mathbb{Z}^{p}$ définie par

$$
\bar{V}_{w}(M)=\bigcap_{L \in \mathcal{U}_{V}} V_{L(w)}^{L}(M) .
$$




\section{R. BAHLOUL}

Comme conséquence du théorème $\mathrm{S} 1$, nous avons le corollaire suivant.

Corollaire S2.

a) Pour tout $w \in \mathbb{Z}^{p}$,

$$
\bar{V}_{w}(M)=\bigcap_{L \in \mathcal{L}(\Sigma)} V_{L(w)}^{L}(M),
$$

où $\mathcal{L}(\Sigma)$ désigne l'ensemble des éléments primitifs du 1-squelette de $\Sigma$.

b) Il existe $\kappa \in \mathbb{N}^{p}$ tel que pour tout $w \in \mathbb{Z}^{p}$, on ait

$$
V_{w}(M) \subset \bar{V}_{w}(M) \subset V_{w+\kappa}(M) .
$$

Faisons quelques commentaires sur la preuve de ce résultat. L'assertion (a) de S2 découle trivialement de S1, en effet

$$
\begin{aligned}
\bar{V}_{w}(M) & =\bigcap_{\sigma \in \Sigma}\left(\bigcap_{L \in \sigma} V_{L(w)}^{L}(M)\right) \\
& =\bigcap_{\sigma \in \Sigma}\left(\bigcap_{L \in \mathcal{L}(\sigma)} V_{L(w)}^{L}(M)\right) \quad(\text { par S1) } \\
& =\bigcap_{L \in \mathcal{L}(\Sigma)} V_{L(w)}^{L}(M) .
\end{aligned}
$$

En ce qui concerne l'assertion (b), Sabbah démontre que si un éventail $\Sigma$ satisfait l'énoncé du théorème S1 alors la filtration $\bar{V}(M)$ est une bonne $V\left(\mathcal{D}_{n+p}\right)$ filtration ce qui, par un lemme usuel de comparaison entre bonnes filtrations fournit les inclusions voulues (il faut noter que la première inclusion de (b) est trivialement vraie et que c'est la deuxième qui nous intéresse).

Finissons le rappel de la preuve. Notons $\delta$ la classe de 1 dans le quotient $M=\mathcal{D}_{n+p} / I$. Posons

$$
b(s)=\prod_{L \in \mathcal{L}(\Sigma)}\left(\prod_{-L(v+\kappa)<k \leqslant 0} b_{L}(L(s)-k)\right) .
$$

Par les assertions (a) et (b) de S2, on constate que

$$
b\left(-\partial_{t} t\right) \delta \in \bar{V}_{-v-\kappa}(M) \subset V_{-v}(M),
$$

ce qui signifie que $b(s) \in \mathcal{B}^{v}(f)$.

Nous allons maintenant énoncer les résultats principaux du présent article.

On se donne un idéal $I$ de $\mathcal{D}_{m}$ (anneau des opérateurs différentiels à coefficients analytiques faisant intervenir $m$ variables). Dans [ACG01], Assi, Castro Jiménez et Granger ont introduit l'ensemble $\mathcal{U}$ des formes linéaires $L$ pour lesquelles la filtration de $\mathcal{D}_{m}$ naturellement associée est compatible avec la structure non commutative de $\mathcal{D}_{m}$ (cf. paragraphe 1.2). Ils ont étudié le comportement du gradué $\operatorname{gr}^{L}(I)$ lorque $L$ varie dans $\mathcal{U}$. Considérons la relation sur $\mathcal{U}$ telle que $L$ et $L^{\prime}$ sont en relation si les gradués $\operatorname{gr}^{L}(h(I))$ et $\operatorname{gr}^{L^{\prime}}(h(I))$ sont égaux $(h(I)$ désigne l'homogénéisé de $I$ dont nous rappelons la définition plus loin). Cette relation est une relation d'équivalence donnant sur $\mathcal{U}$ une partition constituée de cônes polyédraux rationnels convexes. L'eventail ainsi constitué est appelé éventail de Gröbner (analytique) associé à $h(I)$ et noté $\mathcal{E}(h(I))$.

Maintenenant, posons $m=n+p$ et reprenons les notations précédentes. Comme nous le verrons, on peut naturellement inclure $\mathcal{U}_{V}$ dans $\mathcal{U}$. On note alors $\mathcal{E}_{V}=\mathcal{E}_{V}(h(I))$ l'éventail obtenu comme restriction de $\mathcal{E}(h(I))$ à $\mathcal{U}_{V}$. Voici les résultats qu'on se propose de montrer ici. 
ThÉorème 1. Pour tout cône $\sigma$ de $\mathcal{E}_{V}$,

$$
{ }^{\sigma} V_{w}(M)=\bigcap_{L \in \mathcal{L}(\sigma)} V_{L(w)}^{L}(M)
$$

L'analogue du corollaire S2 en découle pour (a) et (b) en remplaçant le 1-squelette de $\Sigma$ par celui de $\mathcal{E}_{V}$. En effet, la preuve du corollaire S2 fonctionne pour n'importe quel éventail dès que ce dernier satisfait le théorème $\mathrm{S} 1$.

ThÉorème 2. Pour $p=2$, il existe $\kappa \in \mathbb{N}^{2}$ calculable à partir de bases de Gröbner associées à chaque cône de l'éventail $\mathcal{E}_{V}$ (ce calcul sera détaillé à la section 3 ) tel que pour tout $w \in \mathbb{Z}^{2}$,

$$
\bar{V}_{w+\kappa}(M) \subset V_{w}(M) .
$$

Pour $p \geqslant 3$, une généralisation de ce résultat semble se heurter à des difficultés techniques difficiles à résoudre.

En résumé, voici l'apport du présent article :

i) Un énoncé et une preuve constructifs du théorème clé S1 ce qui fournit une approche plus élémentaire et plus constructive de l'étape 2 de la preuve de Sabbah et qui permet d'éviter la notion d'éventail adapté.

ii) Pour $p=2$, une preuve entièrement constructive de l'étape 2 dans la preuve de Sabbah.

Je signale que cet article constitue une partie de ma thèse [Bah03] dans laquelle on pourra trouver une autre preuve de l'analogue (i.e. avec $\mathcal{L}\left(\mathcal{E}_{V}\right)$ au lieu de $\mathcal{L}(\Sigma)$ ) de l'assertion (a) de $\mathrm{S} 2$ sans passer par le théorème 1.

Décrivons le contenu de cet article. Dans une première section, nous faisons un certain nombre de rappels concernant le théorème de division dans $\mathcal{D}_{m}\langle z\rangle$ tel qu'il est énoncé dans [ACG01], les notions de base standard et d'éventail de Gröbner. La section 2 est consacrée à la démonstration du théorème 1 . Dans la troisième section, nous démontrons le théorème 2.

\section{Rappels et résultats préparatoires}

Dans les paragraphes qui suivent, nous rappelons, sans donner les démonstrations, un certain nombre de notions et de résultats qui nous seront utiles dans les sections 2 et 3 .

\subsection{Homogénéisation}

Comme dans [CN97], nous introduisons l'anneau $\mathcal{D}_{m}\langle z\rangle$. C'est avec cet anneau que les auteurs de [ACG01] ont introduit l'éventail de Gröbner analytique.

Dans ce paragraphe et jusqu'au paragraphe 1.3.2, $x=\left(x_{1}, \ldots, x_{m}\right)$ et $\mathcal{D}_{m}$ désigne l'anneau des opérateurs différentiels à coefficients dans $\mathbb{C}\{x\}$. On définit l'anneau $\mathcal{D}_{m}\langle z\rangle$ comme la $\mathbb{C}\{x\}$-algèbre engendrée par $\partial_{x_{1}}, \ldots, \partial_{x_{m}}, z$ où les seules relations de commutation non triviales sont

$$
\left[\partial_{x_{i}}, c(x)\right]=\frac{\partial c(x)}{\partial x_{i}} z \quad \text { pour } i=1, \ldots, n \text { et } c(x) \in \mathbb{C}\{x\} .
$$

Dans l'anneau $\mathcal{D}_{m}$, on note $\operatorname{deg}(P)$ le degré total en les $\partial_{x_{i}}$ d'un élément $P$. Considérons la filtration associée. Elle s'étend naturellement à $\mathcal{D}_{m}\langle z\rangle$ en considérant le degré total en les $\partial_{x_{i}}$ et $z$. Cette filtration fait de $\mathcal{D}_{m}\langle z\rangle$ une algèbre graduée :

$$
\mathcal{D}_{m}\langle z\rangle=\bigoplus_{d \in \mathbb{N}} \mathcal{D}_{m}\langle z\rangle_{d} \quad \text { avec } \quad \mathcal{D}_{m}\langle z\rangle_{d}=\bigoplus_{k+|\beta|=d} \mathbb{C}\{x\} \partial_{x}^{\beta} z^{k},
$$




\section{R. BAHLOUL}

où $\beta \in \mathbb{N}^{m}, \partial_{x}^{\beta}=\partial_{x_{1}}^{\beta_{1}} \cdots \partial_{x_{m}}^{\beta_{m}}$ et $|\beta|=\beta_{1}+\cdots+\beta_{m}$. Remarquons que la filtration de $\mathcal{D}_{m}$ donnée par le degré deg donne lieu à un anneau de Rees qui est isomorphe à l'anneau $\mathcal{D}_{m}\langle z\rangle$.

On dit qu'un opérateur $P \in \mathcal{D}_{m}\langle z\rangle$ est homogène (de degré $d$ ) si $P \in \mathcal{D}_{m}\langle z\rangle_{d}$. Pour $P \in \mathcal{D}_{m}$, on définit son homogénéisé $h(P) \in \mathcal{D}_{m}\langle z\rangle$ comme suit: on écrit $P=\sum_{\beta} c_{\beta}(x) \partial_{x}^{\beta}$ et on pose $h(P)=\sum_{\beta} c_{\beta}(x) \partial_{x}^{\beta} z^{d-|\beta|}$ où $d=\operatorname{deg}(P)$, ainsi $h(P)$ est homogène de degré $\operatorname{deg}(P)$. De plus pour un idéal $I$ de $\mathcal{D}_{m}$, on définit $h(I)$ comme l'idéal (à gauche) de $\mathcal{D}_{m}\langle z\rangle$ engendré par l'ensemble des $h(P)$ avec $P$ parcourant $I$.

\subsection{Filtrations, divisions et bases standards}

Nous rappelons les notions usuelles de filtrations sur $\mathcal{D}_{m}$ et $\mathcal{D}_{m}\langle z\rangle$, le théorème de division dans $\mathcal{D}_{m}\langle z\rangle$ de [ACG01]. Nous rappelons aussi les notions de bases standards et bases standards réduites minimales.

Soit $\mathcal{U}$ l'ensemble des formes linéaires $L: \mathbb{R}^{2 m} \rightarrow \mathbb{R}, L(\alpha, \beta)=\sum_{1}^{n} e_{i} \alpha_{i}+\sum_{1}^{n} f_{i} \beta_{i}$ avec pour tout $i=1, \ldots, n, e_{i} \leqslant 0$ et $e_{i}+f_{i} \geqslant 0$. On étend $\mathcal{U}$ à $\mathbb{R}^{2 m+1}$ en posant $L(\alpha, \beta, k)=L(\alpha, \beta)$. Pour $P \in \mathcal{D}_{m}\langle z\rangle$ (respectivement $P \in \mathcal{D}_{m}$ ), on écrit $P=\sum_{\alpha, \beta, k} a_{\alpha, \beta, k} x^{\alpha} \partial_{x}^{\beta} z^{k}$ (avec $a_{\alpha, \beta, k}=0$ pour $k>0$ si $\left.P \in \mathcal{D}_{m}\right)$. On définit le diagramme de Newton de $P$, noté $\mathcal{N}(P) \subset \mathbb{N}^{2 m+1}$ (respectivement $\left.\subset \mathbb{N}^{2 m}\right)$, comme l'ensemble des $(\alpha, \beta, k) \in \mathbb{N}^{2 m+1}$ pour lesquels $a_{\alpha, \beta, k} \neq 0$.

Etant donné $L \in \mathcal{U}$ et $P \in \mathcal{D}_{m}\langle z\rangle$ (ou $P \in \mathcal{D}_{m}$ ), on définit l'ordre de $P$ par rapport à $L$ comme $\operatorname{ord}^{L}(P)=\max L(\mathcal{N}(P))$. Cette ordre induit une filtration $F^{L}$ sur $\mathcal{D}_{m}\langle z\rangle$ ou $\mathcal{D}_{m}$ indexée par $L\left(\mathbb{N}^{2 m+1}\right)$ donnée par

$$
F_{k}^{L}\left(\mathcal{D}_{m}\langle z\rangle\right)=\left\{P \in \mathcal{D}_{m}\langle z\rangle ; \operatorname{ord}^{L}(P) \leqslant k\right\}
$$

ainsi qu'un gradué associé

$$
\operatorname{gr}^{L}\left(\mathcal{D}_{m}\langle z\rangle\right)=\bigoplus_{k \in L\left(\mathbb{N}^{2 m+1}\right)} F_{k}^{L}\left(\mathcal{D}_{m}\langle z\rangle\right) / F_{<k}^{L}\left(\mathcal{D}_{m}\langle z\rangle\right) .
$$

Pour $P \in \mathcal{D}_{m}\langle z\rangle$, on note $\sigma^{L}(P) \in \operatorname{gr}^{L}\left(\mathcal{D}_{n}\langle z\rangle\right)$ le symbole principal de $P$, i.e. la classe de $P$ dans quotient $F_{k}^{L}\left(\mathcal{D}_{m}\langle z\rangle\right) / F_{<k}^{L}\left(\mathcal{D}_{m}\langle z\rangle\right)$ avec $k=\operatorname{ord}^{L}(P)$. Si $J$ est un idéal de $\mathcal{D}_{m}\langle z\rangle$, on a une filtration $F^{L}(J)$ induite qui conduit à un idéal gradué de $\operatorname{gr}^{L}\left(\mathcal{D}_{m}\langle z\rangle\right)$ qu'on note $\operatorname{gr}^{L}(J)$ et qui est engendré par l'ensemble des $\sigma^{L}(P)$ pour $P \in J$.

Pour une forme $L \in \mathcal{U}$, on définit deux ordres, $<_{L}$ sur $\mathbb{N}^{2 m}$ et $<_{L}^{h}$ sur $\mathbb{N}^{2 m+1}$ :

$$
(\alpha, \beta)<_{L}\left(\alpha^{\prime}, \beta^{\prime}\right) \Longleftrightarrow\left\{\begin{array}{l}
L(\alpha, \beta)<L\left(\alpha^{\prime}, \beta^{\prime}\right) \\
\text { ou (égalité et } \left.|\beta|<\left|\beta^{\prime}\right|\right) \\
\text { ou (égalités et } \left.(\alpha, \beta)>_{0}\left(\alpha^{\prime}, \beta^{\prime}\right)\right),
\end{array}\right.
$$

où $<_{0}$ est un bon ordre compatible avec l'addition fixé pour toute la suite ;

$$
(\alpha, \beta, k)<_{L}^{h}\left(\alpha^{\prime}, \beta^{\prime}, k^{\prime}\right) \Longleftrightarrow\left\{\begin{array}{l}
k+|\beta|<k^{\prime}+\left|\beta^{\prime}\right| \\
\text { ou (égalité et } \left.(\alpha, \beta)<_{L}\left(\alpha^{\prime}, \beta^{\prime}\right)\right) .
\end{array}\right.
$$

Pour $P \in \mathcal{D}_{m}\langle z\rangle$, on définit l'exposant privilégié $\exp _{<_{L}^{h}}(P)=\max _{<_{L}^{h}}(\mathcal{N}(P))$ et le monôme privilégié $\operatorname{mp}_{<_{L}^{h}}(P)=\left(x, \partial_{x}, z\right)^{\exp _{<_{L}^{h}}(P)}$. De même pour $P \in \mathcal{D}_{m}$ et $<_{L}$ à la place de $<_{L}^{h}$. Notons que $\exp _{\prec}(P Q)=\exp _{\prec}(P)+\exp _{\prec}(Q)$ dès que $\prec$ est compatible avec l'addition ce qui est le cas pour $<_{L}$ et $<_{L}^{h}$. Rappelons le théorème de division dans $\mathcal{D}_{m}\langle z\rangle$ de [ACG01]. 
Existence De Polynômes DE Bernstein-SATo

Soit $L \in \mathcal{U}$. Soient $Q_{1}, \ldots, Q_{r}$ une famille d'éléments de $\mathcal{D}_{m}\langle z\rangle$. Notons $\left(\Delta_{1}, \ldots, \Delta_{r}, \bar{\Delta}\right)$ la partition de $\mathbb{N}^{2 m+1}$ définie à partir $\operatorname{des} \exp _{<_{L}^{h}}\left(Q_{j}\right)$ :

$$
\begin{aligned}
& \Delta_{1}=\exp _{<_{L}^{h}}\left(Q_{1}\right)+\mathbb{N}^{2 m+1}, \\
& \Delta_{j}=\left(\exp _{<_{L}^{h}}\left(Q_{j}\right)+\mathbb{N}^{2 m+1}\right) \backslash\left(\bigcup_{i=1}^{i=j-1} \Delta_{i}\right) \text { pour } j=2, \ldots, r, \\
& \bar{\Delta}=\mathbb{N}^{2 m+1} \backslash\left(\bigcup_{j=1}^{j=r} \Delta_{j}\right) .
\end{aligned}
$$

ThÉorème 1.1 [ACG01, Theorem 7]. Pour tout $P \in \mathcal{D}_{m}\langle z\rangle$ il existe un unique $\left(q_{1}, \ldots, q_{r}, R\right) \in$ $\left(\mathcal{D}_{m}\langle z\rangle\right)^{r+1}$ tel que :

i) $P=q_{1} Q_{1}+\cdots+q_{r} Q_{r}+R$,

ii) pour tout $j=1, \ldots, r$, si $q_{j} \neq 0$ alors $\mathcal{N}\left(q_{j}\right)+\exp _{<_{L}^{h}}\left(Q_{j}\right) \subset \Delta_{j}$,

iii) si $R \neq 0$ alors $\mathcal{N}(R) \subset \bar{\Delta}$.

On appelle $R$ le reste de la division de $P$ par les $Q_{j}$ relativement $\grave{a}<_{L}^{h}$.

Corollaire 1.2 .

$$
\begin{aligned}
\exp _{<_{L}^{h}}(P) & =\max _{<_{L}^{h}}\left\{\exp _{<_{L}^{h}}\left(q_{j} Q_{j}\right), j=1, \ldots, r ; \exp _{<_{L}^{h}}(R)\right\} \\
\operatorname{ord}^{L}(P) & =\max \left\{\operatorname{ord}^{L}\left(q_{j} Q_{j}\right), j=1, \ldots, r ; \operatorname{ord}^{L}(R)\right\} .
\end{aligned}
$$

Soit $J$ un idéal de $\mathcal{D}_{m}\langle z\rangle$ et $Q_{1}, \ldots, Q_{r} \in J$. On dit que $Q_{1}, \ldots, Q_{r}$ forment une $<_{L}^{h}$-base standard de $J$ si pour tout $P$ dans $J$, le reste de la division de $P$ par $Q_{1}, \ldots, Q_{r}$ est nul. Considérons l'ensemble des exposants de $J, \operatorname{Exp}_{<_{L}^{h}}(J)=\left\{\exp _{<_{L}^{h}}(P), P \in J \backslash 0\right\}$. Etant donnés $Q_{1}, \ldots, Q_{r} \in J$, les deux assertions suivantes sont équivalentes (grâce aux théorème de division) :

a) Les $Q_{j}$ forment une $<_{L}^{h}$-base standard de $J$.

b) $\operatorname{Exp}_{<_{L}^{h}}(J)=\bigcup_{j=1}^{r}\left(\exp _{<_{L}^{h}}\left(Q_{j}\right)+\mathbb{N}^{2 m+1}\right)$.

L'existence d'une base standard est assurée par le lemme de Dickson qui dit que si une partie $E$ de $\mathbb{N}^{q}\left(q \in \mathbb{N} \backslash 0\right.$ ) vérifie $E+\mathbb{N}^{q}=E$ (ce qui est le cas de $\left.\operatorname{Exp}_{<_{L}^{h}}(J)\right)$ alors il existe $F \subset E$ fini tel que $E=\bigcup_{e \in F}\left(e+\mathbb{N}^{q}\right)$.

Il est facile de voir qu'une base standard n'est pas unique, c'est pour cela qu'il existe une notion de base standard réduite minimale.

DÉfinition. Soit $Q_{1}, \ldots, Q_{r}$ une $<_{L}^{h}$-base standard de $J \subset \mathcal{D}_{m}\langle z\rangle$ et soit $e_{j}=\exp _{<_{L}^{h}}\left(Q_{j}\right)$ pour $j=1, \ldots, r$.

i) On dit qu'elle est minimale si pour toute partie finie $F$ de $\mathbb{N}^{2 m+1}$ on a l'implication suivante :

$$
\operatorname{Exp}_{<_{L}^{h}}(J)=\bigcup_{e \in F}\left(e+\mathbb{N}^{2 m+1}\right) \Rightarrow\left\{e_{1}, \ldots, e_{r}\right\} \subseteq F .
$$

ii) On dit qu'elle est réduite si les $Q_{j}$ sont unitaires (i.e. le coefficient du monône privilégié est 1) et si pour tout $j$

$$
\left(\mathcal{N}\left(Q_{j}\right) \backslash e_{j}\right) \subset\left(\mathbb{N}^{2 m+1} \backslash \operatorname{Exp}_{<L}(J)\right) .
$$

Il existe une unique $<_{L}^{h}$-base standard réduite minimale d'un idéal $J \subset \mathcal{D}_{m}\langle z\rangle$. 


\section{R. BAHLOUL}

Notons que si l'idéal $J$ est homogène alors la base standard réduite minimale est constituée d'éléments homogènes. Nous terminons ce paragraphe par un lemme qui illustre l'intérêt des bases standards réduites minimales. Nous l'appliquerons dans la section suivante.

Lemme 1.3. Soient $<_{1}$ et $<_{2}$ deux ordres sur $\mathbb{N}^{2 m+1}$ qui permettent de faire des divisions dans $\mathcal{D}_{m}\langle z\rangle$ (par exemple $<_{i}=<_{L_{i}}^{h}$ avec $L_{1}$ et $L_{2}$ deux formes). Soit $\left\{Q_{1}, \ldots, Q_{r}\right\}$ la base standard réduite minimale d'un idéal $J \subset \mathcal{D}_{m}\langle z\rangle$ par rapport à l'ordre $<_{1}$. Supposons que pour tout $j, \exp _{<_{1}}\left(Q_{j}\right)$ et $\exp _{<_{2}}\left(Q_{j}\right)$ soient égaux. Alors $\left\{Q_{1}, \ldots, Q_{r}\right\}$ est aussi la base standard réduite minimale de $J$ par rapport à $<_{2}$.

Nous omettons la preuve. Disons simplement que ce qui importe lors d'une division, c'est l'ensemble des $\exp _{<}\left(Q_{j}\right)$ et pas l'ordre $<$. Ainsi, diviser par les $Q_{j}$ par rapport à $<_{1}$ ou $<_{2}$ donnera les mêmes quotients et le même reste.

\subsection{Eventail de Gröbner}

Dans ce paragraphe, nous rappelons le résultat principal de [ACG01] décrivant l'éventail de Gröbner d'un idéal. Nous introduisons aussi le $V$-éventail de Gröbner $\mathcal{E}_{V}(h(I))$; ce sera l'objet principal des sections 2 et 3 .

1.3.1 On se donne un idéal $I$ de $\mathcal{D}_{m}$ et on considère $h(I) \subset \mathcal{D}_{m}\langle z\rangle$ son homogénéisé. Pour $L$ et $L^{\prime}$ dans $\mathcal{U}$, on définit la relation

$$
L \sim L^{\prime} \Longleftrightarrow \operatorname{gr}^{L}\left(\mathcal{D}_{m}\langle z\rangle\right)=\operatorname{gr}^{L^{\prime}}\left(\mathcal{D}_{m}\langle z\rangle\right) \text { et } \operatorname{gr}^{L}(h(I))=\operatorname{gr}^{L^{\prime}}(h(I)) .
$$

Cette relation est une relation d'équivalence sur $\mathcal{U}$.

ThÉorème 1.4 [ACG01]. La partition sur $\mathcal{U}$ donnée par la relation d'équivalence précédente est constituée de cônes polyédraux rationnels convexes. Cette partition qu'on note $\mathcal{E}(h(I))$ est appelée éventail de Gröbner (analytique) de $h(I)$.

De plus pour chaque cône $\sigma \in \mathcal{E}(h(I))$, il existe $Q_{1}, \ldots, Q_{r} \in h(I)$ homogènes tels que

i) pour tout $L, L^{\prime} \in \sigma, \sigma^{L}\left(Q_{j}\right)=\sigma^{L^{\prime}}\left(Q_{j}\right)$ et $\exp _{<_{L}^{h}}\left(Q_{j}\right)=\exp _{<_{L^{\prime}}}\left(Q_{j}\right)$ pour tout $j$;

ii) pour tout $L \in \sigma$, l'ensemble $\left\{Q_{1}, \ldots, Q_{r}\right\}$ est la base standard réduite minimale de $h(I)$ par rapport à $<_{L}^{h}$.

Remarquons que la deuxième condition nous montre que sur un cône $\sigma$, l'ensemble $\operatorname{Exp}_{<_{L}^{h}}(h(I))$ des exposants de $h(I)$ par rapport à $<_{L}^{h}$ est constant lorsque $L$ parcourt $\sigma$.

1.3.2 A partir de maintenant, on travaillera dans $\mathcal{D}_{n+p}$ et $\mathcal{D}_{n+p}\langle z\rangle$ (i.e. $m=n+p$ ). Pour $j=1, \ldots, p$, on notera $V_{j} \in \mathcal{U}$ la forme linéaire donnée par $V_{j}(\alpha, \mu, \beta, \nu)=\nu_{j}-\mu_{j}$ où $\alpha, \beta \in \mathbb{N}^{n}$ et $\mu, \nu \in \mathbb{N}^{p}$. Cette forme linéaire donne lieu à une filtration qu'on note aussi $V_{j}$ et qui n'est rien d'autre que la $V$-filtration de Kashiwara-Malgrange associée à la variable $t_{j}$ (rappelons que dans $\mathcal{D}_{n+p}$, les variables sont $x=\left(x_{1}, \ldots, x_{n}\right)$ et $\left.t=\left(t_{1}, \ldots, t_{p}\right)\right)$. On note alors $V$ la multifiltration $V=\left(V_{1}, \ldots, V_{p}\right)$.

Nous noterons $\mathcal{U}_{V} \subset \mathcal{U}$ le sous-ensemble des formes linéaires $L$ s'écrivant

$$
L=l_{1} V_{1}+\cdots+l_{p} V_{p},
$$

avec $\left(l_{1}, \ldots, l_{p}\right) \in\left(\mathbb{R}_{\geqslant 0}\right)^{p}$, aussi nous identifierons $\mathcal{U}_{V}$ et $\left(\mathbb{R}_{\geqslant 0}\right)^{p}$.

A partir de maintenant, pour $L \in \mathcal{U}_{V}$, on notera $V^{L}$ la filtration associée à $L$ (conformément aux notations de [Sab87a]). Notons que pour $L \in \mathcal{U}_{V}, \operatorname{gr}^{L}\left(\mathcal{D}_{n+p}\langle z\rangle\right)$ s'identifie à un sous-anneau de $\mathcal{D}_{n+p}\langle z\rangle$. En effet, quitte à réindexer les variables, supposons que $L=l_{1} V_{1}+\cdots+l_{e} V_{e}$ avec $0 \leqslant e \leqslant p$ 


\section{Existence De POLYNÔMES DE BeRnstein-SATO}

et qu'aucun des $l_{j}$ n'est nul (par convention, si $e=0$ alors $L=0$ et $\operatorname{gr}^{L}\left(\mathcal{D}_{n+p}\langle z\rangle\right)=\mathcal{D}_{n+p}\langle z\rangle$ ). Dans ce cas, nous avons

$$
\operatorname{gr}^{L}\left(\mathcal{D}_{n+p}\langle z\rangle\right)=\mathbb{C}\left\{x_{1}, \ldots, x_{n}, t_{e+1}, \ldots, t_{p}\right\}\left[t_{1}, \ldots, t_{e}\right]\left[\partial_{x_{1}}, \ldots, \partial_{x_{n}}, \partial_{t_{1}}, \ldots, \partial_{t_{p}}\right]\langle z\rangle,
$$

dans lequel les relations de commutation coïncident avec celles de $\mathcal{D}_{n+p}\langle z\rangle$. Ainsi nous considérerons que tous les calculs se feront dans $\mathcal{D}_{n+p}\langle z\rangle$. Maintenant, considérons la restriction de $\mathcal{E}(h(I))$ à $\mathcal{U}_{V}$. Ceci donne lieu au $V$-éventail de Gröbner de $h(I)$ que l'on note $\mathcal{E}_{V}(h(I))$. Comme conséquence, on obtient un analogue du théorème 1.4.

Corollaire 1.5. Pour chaque cône $\sigma$ de $\mathcal{E}_{V}(h(I))$, il existe $Q_{1}, \ldots, Q_{r} \in h(I)$ homogènes tels que

i) pour toute $L, L^{\prime} \in \sigma, \sigma^{L}\left(Q_{j}\right)=\sigma^{L^{\prime}}\left(Q_{j}\right)$ et $\exp _{<_{L}^{h}}\left(Q_{j}\right)=\exp _{<_{L^{\prime}}^{h}}\left(Q_{j}\right)$ pour tout $j$;

ii) pour tout $L \in \sigma$, l'ensemble $\left\{Q_{1}, \ldots, Q_{r}\right\}$ est la base standard réduite minimale de $h(I)$ par rapport à $<_{L}^{h}$.

On voit que pour chaque cône $\sigma$, il existe des $Q_{j}$ qui, pour tout $L$ de $\sigma$, forment la base standard réduite minimale de $h(I)$ par rapport à $<_{L}^{h}$. S'il n'y pas de confusion possible, on l'appelle la base standard de $h(I)$ associée à $\sigma$.

Terminons ce paragraphe par quelques remarques et notations. Un cône $\sigma$ de $\mathcal{E}_{V}(h(I))$ n'est pas nécessairement ouvert (il peut être 'semi-ouvert'). Voici comment on définit $\mathcal{L}(\sigma)$ : On considère d'abord l'adhérence $\bar{\sigma}$ de $\sigma$. Il existe $L_{1}, \ldots, L_{q} \in \mathcal{U}_{V}$ qu'on suppose primitifs (avec $q \geqslant 1$ pouvant être plus grand que $p$ ) tels que

$$
\bar{\sigma}=\left\{L=r_{1} L_{1}+\cdots+r_{q} L_{q} ; r_{i} \geqslant 0\right\} .
$$

Supposons $q$ minimal alors $\mathcal{L}(\sigma)$ est l'ensemble $\left\{L_{1}, \ldots, L_{q}\right\}$. L'ensemble $\mathcal{L}\left(\mathcal{E}_{V}(h(I))\right)$ n'est alors rien d'autre que la réunion des $\mathcal{L}(\sigma)$ avec $\sigma \in \mathcal{E}_{V}(h(I))$ (remarquons qu'il est constitué d'éléments entiers car tout cône $\sigma$ est rationnel).

On définit l'intérieur de $\sigma$ comme l'ensemble des combinaisons à coefficients strictement positifs des $L_{i}$. On note $\rangle L_{1}, \ldots, L_{q}\left\langle\right.$ le cône ouvert engendré par les $L_{i}$. D'autre part si $L_{1}$ et $L_{2}$ sont dans $\mathcal{U}_{V}$, on note $\left.\rangle L_{1}, L_{2}\right\rangle=\left\{r_{1} L_{1}+r_{2} L_{2} ; r_{1}>0, r_{2} \geqslant 0\right\}$ le cône semi-ouvert contenant $L_{2}$ mais non $L_{1}$.

Dans la suite, on écrira $\mathcal{E}_{V}$ à la place de $\mathcal{E}_{V}(h(I))$.

\section{Démonstration du théorème 1}

Soit $\sigma$ un cône de $\mathcal{E}_{V}$. Nous avons vu dans le corollaire 1.5 qu'il existe une famille $Q_{1}, \ldots, Q_{r}$ de $h(I)$ qui est la base standard réduite minimale de $h(I)$ par rapport à $<_{L}^{h}$ et ce pour tout $L$ dans $\sigma$. Maintenant, que se passe-t-il pour une forme linéaire $L$ appartenant à l'adhérence de $\sigma$ mais qui n'est pas dans $\sigma$ (ce qui peut arriver pour un élément de $\mathcal{L}(\sigma)$ ) ? Bien entendu les $Q_{j}$ ne forment pas une base standard de $h(I)$ pour l'ordre $<_{L}^{h}$. Par contre il est possible, et c'est le but du résultat suivant, de construire un ordre que nous noterons $\triangleleft_{L}^{\sigma}$ (car il dépend de $L$ et de $\sigma$ ) pour lequel les $Q_{j}$ forment la base standard réduite minimale de $h(I)$. Voici comment on définit l'ordre en question.

D'abord on fixe une forme linéaire $L_{\sigma}$ dans l'intérieur de $\sigma$ et pour $(\alpha, \mu, \beta, \nu, k)$ et $\left(\alpha^{\prime}, \mu^{\prime}, \beta^{\prime}\right.$, $\left.\nu^{\prime}, k^{\prime}\right)$ dans $\mathbb{N}^{n+p+n+p+1}$ on pose

$$
(\alpha, \mu, \beta, \nu, k) \triangleleft_{L}^{\sigma}\left(\alpha^{\prime}, \mu^{\prime}, \beta^{\prime}, \nu^{\prime}, k\right) \Longleftrightarrow\left\{\begin{array}{l}
k+|\beta|+|\nu|<k^{\prime}+\left|\beta^{\prime}\right|+\left|\nu^{\prime}\right| \\
\text { ou }\left(=\text { et } L(\alpha, \mu, \beta, \nu)<L\left(\alpha^{\prime}, \mu^{\prime}, \beta^{\prime}, \nu^{\prime}\right)\right) \\
\text { ou }\left(=\text { et }=\text { et }(\alpha, \mu, \beta, \nu)<_{L_{\sigma}}\left(\alpha^{\prime}, \mu^{\prime}, \beta^{\prime}, \nu^{\prime}\right)\right) .
\end{array}\right.
$$




\section{R. BAHLOUL}

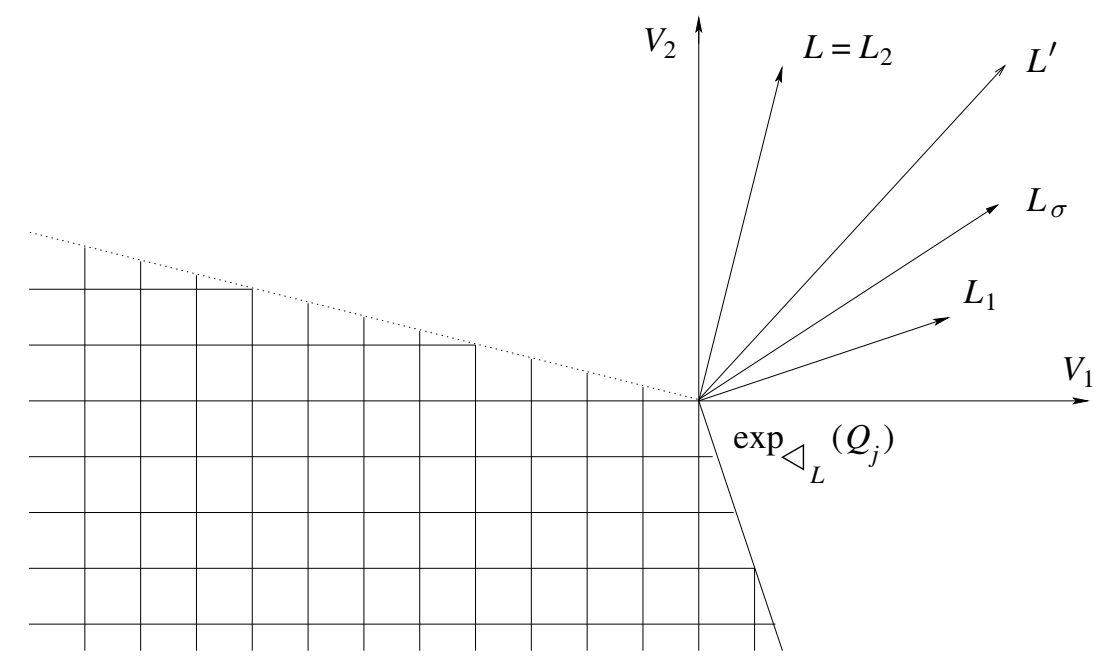

Figure 2. Diagramme de Newton d'un $Q_{j}$ associé à $\left.\left.\sigma=\right\rangle L_{2}, L_{1}\right\rangle$.

Proposition 2.1. Soit $L \in \mathcal{U}_{V}$ appartenant à l'adhérence de $\sigma \in \mathcal{E}_{V}$ et soit $Q_{1}, \ldots, Q_{r}$ la base standard de $h(I)$ associée au cône $\sigma$. Alors pour tout $j=1, \ldots, r$ et pour tout $L^{\prime}$ dans $\left.\rangle L, L_{\sigma}\right\rangle$

$$
\exp _{<_{L^{\prime}}^{h}}\left(Q_{j}\right)=\exp _{\triangleleft_{L}^{\sigma}}\left(Q_{j}\right) \text {. }
$$

Comme conséquence de cette proposition, nous obtenons que les $Q_{j}$ forment la base standard réduite minimale de $h(I)$ pour l'ordre $\triangleleft_{L}^{\sigma}$. En effet, il suffit pour cela d'appliquer le lemme 1.3.

Démonstration. Pour la commodité du lecteur, la figure 2 illustre la situation de la proposition (avec $p=2$, mais en dimension supérieure, on pourrait faire une figure similaire en intersectant $\mathcal{U}_{V}$ avec le plan engendré par $L$ et $\left.L_{\sigma}\right)$. Nous avons :

$$
\begin{aligned}
\exp _{\triangleleft_{L}^{\sigma}}\left(Q_{j}\right) & =\exp _{\triangleleft_{L}^{\sigma}}\left(\sigma^{L}\left(Q_{j}\right)\right) \quad\left(\text { par définition de } \triangleleft_{L}^{\sigma} \text { et par homogénéité de } Q_{j}\right) \\
& \left.=\exp _{<_{L_{\sigma}}^{h}}\left(\sigma^{L}\left(Q_{j}\right)\right) \quad \text { (par définition de } \triangleleft_{L}^{\sigma} \text { et }<_{L_{\sigma}}^{h}\right) \\
& =\exp _{<_{L_{\sigma}}^{h}}\left(Q_{j}\right) \quad(\text { par l'affirmation ci-après }) \\
& =\exp _{<_{L^{\prime}}^{h}}\left(Q_{j}\right) \quad(\text { par le corollaire 1.5). }
\end{aligned}
$$

Pour clore la démonstration, il ne reste plus qu'à montrer l'égalité suivante.

Affirmation. Nous avons

$$
\exp _{<_{L_{\sigma}}^{h}}\left(\sigma^{L}\left(Q_{j}\right)\right)=\exp _{<_{L_{\sigma}}^{h}}\left(Q_{j}\right) .
$$

Puisque $\mathcal{N}\left(\sigma^{L}\left(Q_{j}\right)\right) \subseteq \mathcal{N}\left(Q_{j}\right)$, nous avons $\exp _{<_{L_{\sigma}}^{h}}\left(\sigma^{L}\left(Q_{j}\right)\right) \leqslant_{L_{\sigma}}^{h} \exp _{<_{L_{\sigma}}^{h}}\left(Q_{j}\right)$. L'inégalité inverse suffit donc à démontrer l'affirmation. Commençons par montrer l'égalité

$$
\operatorname{ord}^{L}\left(Q_{j}\right)=L\left(\exp _{<_{L_{\sigma}}^{h}}\left(Q_{j}\right)\right) \text {. }
$$

Pour tout $L^{\prime \prime}$ dans $\left.\rangle L, L_{\sigma}\right\rangle$, on a

$$
\operatorname{ord}^{L^{\prime \prime}}\left(Q_{j}\right)=L^{\prime \prime}\left(\exp _{<_{L^{\prime \prime}}^{h}}\left(Q_{j}\right)\right)=L^{\prime \prime}\left(\exp _{<_{L_{\sigma}}^{h}}\left(Q_{j}\right)\right)
$$

par définition de $<_{L^{\prime \prime}}^{h}$ et par le corollaire 1.5. Ainsi pour tout $m$ fixé dans $\mathcal{N}\left(Q_{j}\right)$,

$$
L^{\prime \prime}\left(\exp _{<_{L_{\sigma}}^{h}}\left(Q_{j}\right)\right) \geqslant L^{\prime \prime}(m) .
$$




\section{Existence De POLYNÔMES DE BeRnstein-SATO}

Ecrivons $L^{\prime \prime}=(1-\varepsilon) L+\varepsilon L_{\sigma}$ (avec $1 \geqslant \varepsilon>0$ ) et faisons tendre $\varepsilon$ vers 0 . Par continuité, l'inégalité précédente se conserve à la limite ce qui donne : $L\left(\exp _{<_{L_{\sigma}}^{h}}\left(Q_{j}\right)\right) \geqslant L(m)$, ceci pour tout $m$ dans $\mathcal{N}\left(Q_{j}\right)$. L'egalité $(\star \star)$ est démontrée. En conséquence et par définition de $\sigma^{L}\left(Q_{j}\right)$, nous avons

$$
\exp _{<_{L_{\sigma}}^{h}}\left(Q_{j}\right) \in \mathcal{N}\left(\sigma^{L}\left(Q_{j}\right)\right) .
$$

L'inégalité recherchée découle alors immédiatement de la définition de $\exp _{<_{L_{\sigma}}^{h}}\left(\sigma^{L}\left(Q_{j}\right)\right)$. L'affirmation est démontrée.

Rappelons que $I$ est un idéal de $\mathcal{D}_{n+p}, M=\mathcal{D}_{n+p} / I$ et $\delta$ est la classe de 1 dans $M$. Soit $\sigma$ un cône de $\mathcal{E}_{V}$. Notons $L_{1}, \ldots, L_{q}$ les éléments de $\mathcal{L}(\sigma)$.

Lemme 2.2. Soit $i_{0} \in\{1, \ldots, q\}, m \in V_{\lambda_{i_{0}}}^{L_{i_{0}}}(M)$ avec $\lambda_{i_{0}} \in \mathbb{Q}$ et $P \in \mathcal{D}_{n+p}$ tel que $P \delta=m$ et $\operatorname{ord}^{L_{i}}(P)>\lambda_{i_{0}}$, alors il existe $P^{\prime} \in \mathcal{D}_{n+p}$ tel que

i) $P-P^{\prime} \in I$ c'est-à-dire $P^{\prime} \delta=m$,

ii) $\operatorname{ord}^{L_{i_{0}}}\left(P^{\prime}\right)<\operatorname{ord}^{L_{i_{0}}}(P)$,

iii) $\operatorname{ord}^{L_{i}}\left(P^{\prime}\right) \leqslant \operatorname{ord}^{L_{i}}(P)$ pour $i \in\{1, \ldots, q\} \backslash\left\{i_{0}\right\}$.

En d'autres termes, il est possible de faire baisser l'ordre par rapport à l'un des $L_{i}$ sans augmenter l'ordre par rapport aux autres $L_{i}$. Avec ce lemme, nous sommes en mesure de donner une démonstration du théorème 1

Démonstration du théorème 1. Soit $m \in \bigcap_{L \in \mathcal{L}(\sigma)} V_{L(v)}^{L}(M)$, alors pour $i=1, \ldots, q$ il existe $P_{i} \in$ $\mathcal{D}_{n+p}$ tel que $P_{i} \delta=m$ et $\operatorname{ord}^{L_{i}}\left(P_{i}\right) \leqslant L_{i}(v)$. On pose $\tilde{P}_{1}=P_{1}$. En appliquant un nombre fini de fois le lemme avec $i_{0}=2$ (la première fois avec $P=\tilde{P}_{1}$ et $\lambda_{i_{0}}=\operatorname{ord}^{L_{i}}\left(\tilde{P}_{1}\right)$ ), on construit $\tilde{P}_{2}$ tel que $\tilde{P}_{2} \delta=m$ et $\operatorname{ord}^{L_{i}}\left(\tilde{P}_{2}\right) \leqslant L_{i}(v)$ pour $i=1,2$. On recommence le processus avec $i_{0}=3$ et $P=\tilde{P}_{2}$, etc. Après un nombre fini d'étapes on obtient $\tilde{P}_{q} \in \mathcal{D}_{n+p}$ tel que $\tilde{P}_{q} \delta=m$ et pour tout $i=1, \ldots, q$, $\operatorname{ord}^{L_{i}}\left(\tilde{P}_{q}\right) \leqslant L_{i}(v)$. Ceci montre bien que $m \in{ }^{\sigma} V_{v}(M)$.

Pour finir cette section, il ne reste plus qu’à démontrer le lemme précédent.

Démonstration du lemme 2.2. Pour simplifier les notations, nous ferons la démonstration avec $i_{0}=1$ et nous poserons $\lambda=\lambda_{i_{0}}$ et $\triangleleft_{L_{i}}=\triangleleft_{L_{i}}^{\sigma}$. Notons $Q_{1}, \ldots, Q_{r}$ la base standard de $h(I)$ associée au cône $\sigma$.

Par hypothèse, il existe $P_{1} \in \mathcal{D}_{n+p}$ avec $P_{1} \delta=m$ et $\operatorname{ord}^{L_{1}}\left(P_{1}\right) \leqslant \lambda$. Il existe $l_{0}, l, l_{1} \in \mathbb{N}$ tels que $z^{l_{0}} h\left(P-P_{1}\right)=z^{l} h(P)-z^{l_{1}} h\left(P_{1}\right)$. On pose alors $H=z^{l} h(P), H_{1}=z^{l_{1}} h\left(P_{1}\right)$ et $H_{0}=H-H_{1}$ et comme $P-P_{1} \in I$, on a $H_{0} \in h(I)$.

Considérons la division de $H_{0}$ par les $Q_{j}$ relativement à l'ordre $\triangleleft_{L_{1}}$ :

$$
H_{0}=\sum_{j=1}^{r} q_{j} Q_{j} \quad \text { avec } \mathcal{N}\left(q_{j}\right)+\exp _{\triangleleft_{L_{1}}}\left(Q_{j}\right) \subset \Delta_{j} \text { pour tout } j,
$$

où les $\Delta_{j} \subset \mathbb{N}^{2 n+2 p+1}$ forment la partition de $\operatorname{Exp}_{\triangleleft_{L_{1}}}(h(I))$ associée aux exposants privilégiés des $Q_{j}$ (voir le théorème de division 1.1 rappelé à la section 1 ).

Comme pour tout $i, j$, les exposants $\exp _{\triangleleft_{L_{1}}}\left(Q_{j}\right)$ et $\exp _{\triangleleft_{L_{i}}}\left(Q_{j}\right)$ sont égaux, la division précédente est aussi une division relativement aux ordres $\triangleleft_{L_{2}}, \ldots, \triangleleft_{L_{q}}$. Par conséquent, pour tout $i=1, \ldots, q$ et $j=1, \ldots, r$,

$$
\operatorname{ord}^{L_{i}}\left(H_{0}\right) \geqslant \operatorname{ord}^{L_{i}}\left(q_{j} Q_{j}\right)
$$




\section{R. BAHLOUL}

Notons $J$ l'ensemble des $j \in\{1, \ldots, r\}$ pour lesquels $\operatorname{ord}^{L_{1}}\left(H_{0}\right)=\operatorname{ord}^{L_{1}}\left(q_{j} Q_{j}\right)$, nous avons alors

$$
\sigma^{L_{1}}\left(H_{0}\right)=\sigma^{L_{1}}(H)=\sum_{j \in J} \sigma^{L_{1}}\left(q_{j}\right) \sigma^{L_{1}}\left(Q_{j}\right) .
$$

On considère et on note

$$
W=\sum_{j \in J} \sigma^{L_{1}}\left(q_{j}\right) Q_{j}
$$

C'est un élément de $h(I)$.

Posons $H^{\prime}=H-W$. Nous allons montrer les deux assertions suivantes :

i) $\operatorname{ord}^{L_{1}}\left(H^{\prime}\right)<\operatorname{ord}^{L_{1}}(H)$,

ii) $\operatorname{ord}^{L_{i}}\left(H^{\prime}\right) \leqslant \operatorname{ord}^{L_{i}}(H)$ pour $i=2, \ldots, q$.

Pour montrer l'assertion i, notons qu'on a clairement $\sigma^{L_{1}}(H)=\sigma^{L_{1}}(W)$. Par conséquent,

$$
H^{\prime}=\left(H-\sigma^{L_{1}}(H)\right)-\left(W-\sigma^{L_{1}}(W)\right) .
$$

On voit alors facilement que les deux termes entre parenthèses ont un $L_{1}$-ordre strictement inférieur à celui de $H$.

Pour montrer l'assertion ii, fixons $i$ entre 2 et $q$. En utilisant 1.5, pour tout $j$, on a

$$
\exp _{\triangleleft_{L_{i}}}\left(\sigma^{L_{1}}\left(Q_{j}\right)\right)=\exp _{\triangleleft_{L_{i}}}\left(Q_{j}\right)
$$

D'autre part, par construction des $q_{j}$, pour tout $j$ on a $\mathcal{N}\left(q_{j}\right)+\exp _{\triangleleft_{L_{i}}}\left(Q_{j}\right) \subset \Delta_{j}$, ce qui entraine ceci pour tout $j$ :

$$
\mathcal{N}\left(\sigma^{L_{1}}\left(q_{j}\right)\right)+\exp _{\triangleleft_{L_{i}}}\left(\sigma^{L_{1}}\left(Q_{j}\right)\right) \subset \Delta_{j} .
$$

Maintenant, nous avons $\sigma^{L_{1}}(W)=\sum_{j \in J} \sigma^{L_{1}}\left(q_{j}\right) \sigma^{L_{1}}\left(Q_{j}\right)$. Par la relation (2), on peut dire que cette écriture est le résultat de la division de $\sigma^{L_{1}}(W)$ par $\left\{\sigma^{L_{1}}\left(Q_{j}\right), j \in J\right\}$ relativement à $\triangleleft_{L_{i}}$, par conséquent

$$
\exp _{\triangleleft_{L_{i}}}\left(\sigma^{L_{1}}(W)\right)=\max _{j \in J}\left\{\exp _{\triangleleft_{L_{i}}}\left(\sigma^{L_{1}}\left(q_{j}\right) \sigma^{L_{1}}\left(Q_{j}\right)\right)\right\} .
$$

De la même manière, on peut montrer que

$$
\exp _{\triangleleft L_{i}}(W)=\max _{j \in J}\left\{\exp _{\triangleleft_{L_{i}}}\left(\sigma^{L_{1}}\left(q_{j}\right) Q_{j}\right)\right\} .
$$

Par conséquent, grâce à (3), (4) et (1), on obtient l'égalité $\exp _{\triangleleft_{L_{i}}}\left(\sigma^{L_{1}}(W)\right)=\exp _{\triangleleft_{L_{i}}}(W)$, ce qui donne en particulier $\operatorname{ord}^{L_{i}}(W)=\operatorname{ord}^{L_{i}}\left(\sigma^{L_{1}}(W)\right)$. D'où les égalités et inégalités suivantes :

$$
\begin{aligned}
\operatorname{ord}^{L_{i}}(W) & =\operatorname{ord}^{L_{i}}\left(\sigma^{L_{1}}(W)\right) \\
& =\operatorname{ord}^{L_{i}}\left(\sigma^{L_{1}}(H)\right) \quad\left(\operatorname{car} \sigma^{L_{1}}(W)=\sigma^{L_{1}}(H)\right) \\
& \leqslant \operatorname{ord}^{L_{i}}(H) .
\end{aligned}
$$

Par conséquent, $\operatorname{ord}^{L_{i}}\left(H^{\prime}\right) \leqslant \operatorname{ord}^{L_{i}}(H)$. Les deux assertions sont démontrées.

Maintenant, effectuons la spécialisation $z=1$ (qui est un morphisme d'algèbres $\mathcal{D}_{n+p}\langle z\rangle \rightarrow \mathcal{D}_{n+p}$ ) et posons $P^{\prime}=H_{\mid z=1}^{\prime}=P-W_{\mid z=1}$. On a $W \in h(I)$ donc $W_{\mid z=1} \in I$ et $P^{\prime} \delta=m$. Après spécialisation, les assertions $\mathrm{i}$ et ii deviennent comme suit $: \operatorname{ord}^{L_{i}}\left(P^{\prime}\right) \leqslant \operatorname{ord}^{L_{i}}(P)$ pour tout $i=1, \ldots, q$ avec inégalité stricte pour $i=1$. Le lemme est démontré. 


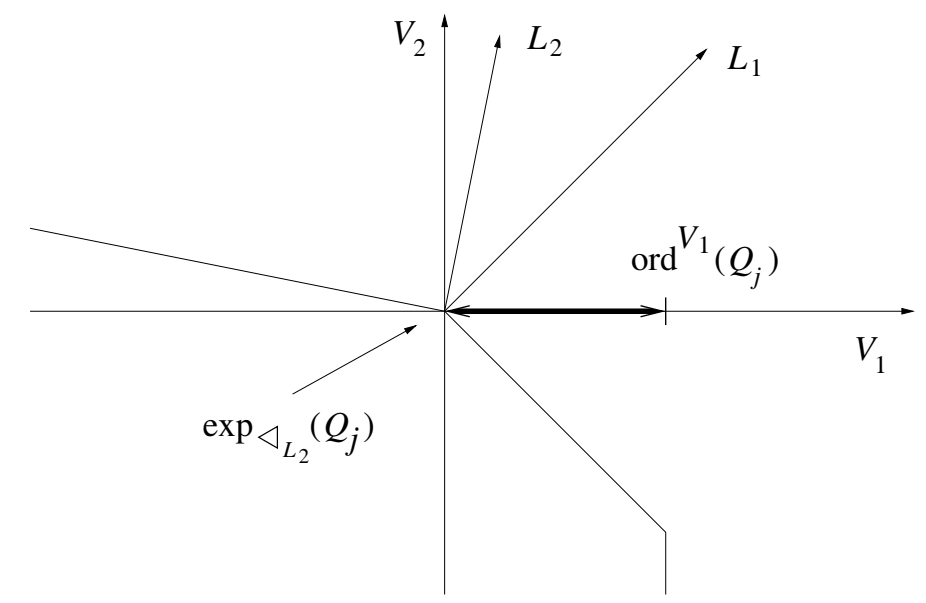

FiguRE 3. La différence $\operatorname{ord}^{V_{1}}\left(Q_{j}\right)-\operatorname{ord}^{V_{1}}\left(\exp _{\triangleleft_{L_{2}}}\left(Q_{j}\right)\right)$.

\section{Démonstration du théorème 2}

Dans cette section, nous allons démontrer le théorème 2. Nous allons dans un premier temps énoncer plus précisément l'assertion en question, puis nous en écrirons la preuve. Nous verrons qu'elle consiste essentiellement en une analyse raffinée du lemme précédent 2.2, en effet nous ferons ce qu'on pourrait appeler un 'contrôle de la montée de l'ordre' par rapport à la forme $V_{1}$.

Rappelons que dans ce paragraphe, $p$ égale 2.

\section{Notation.}

a) Soient $L_{1}, L_{2}$ deux formes non nulles de $\mathcal{U}_{V}$. Ecrivons $L_{i}=a_{i} V_{1}+b_{i} V_{2}$ avec $a_{i}, b_{i} \geqslant 0$. On dit que $L_{1}$ est inférieure (respectivement strictement inférieure) à $L_{2}$ si $b_{1} / a_{1} \leqslant b_{2} / a_{2}$ (respectivement $\left.b_{1} / a_{1}<b_{2} / a_{2}\right)$. On abrègera cette notion en notant $L_{1} \leqslant L_{2}$ (respectivement $L_{1}<L_{2}$ ). Par convention $b / 0=+\infty$, toute forme $L$ est inférieure à $V_{2}$.

b) Soient $L_{1} \neq L_{2}$ dans $\mathcal{U}_{V}$ et $H \in \mathcal{D}_{n+p}\langle z\rangle$. On dit que $H$ est $L_{1}$-homogène si $H=\sigma^{L_{1}}(H)$. On dit que $H$ est $\left(L_{1}, L_{2}\right)$-homogène si $H=\sigma^{L_{1}}\left(\sigma^{L_{2}}(H)\right)$.

c) Soit $L$ une forme dans $\mathcal{U}_{V}$. Nous noterons $\triangleleft_{L}$ l'ordre sur $\mathbb{N}^{n+2+n+2+1}$ donné par

$$
(\alpha, \mu, \beta, \nu, k) \triangleleft_{L}\left(\alpha^{\prime}, \mu^{\prime}, \beta^{\prime}, \nu^{\prime}, k\right) \Longleftrightarrow\left\{\begin{array}{l}
k+|\beta+\nu|<k^{\prime}+\left|\beta^{\prime}+\nu^{\prime}\right| \\
\text { ou }\left(=\text { et } L(\alpha, \mu, \beta, \nu)<L\left(\alpha^{\prime}, \mu^{\prime}, \beta^{\prime}, \nu^{\prime}\right)\right) \\
\text { ou }\left(=\text { et }=\text { et }(\alpha, \mu, \beta, \nu)<_{V_{1}}\left(\alpha^{\prime}, \mu^{\prime}, \beta^{\prime}, \nu^{\prime}\right)\right) .
\end{array}\right.
$$

Nous remarquons qu'en adoptant les notations du paragraphe précédent et en posant $\sigma=$ \rangle $V_{1}, L\left\langle\left(\operatorname{avec} L \neq V_{1}\right)\right.$ alors on a $: \triangleleft_{L}=\triangleleft_{L}^{\sigma}$. Si par contre $L=V_{1}$ alors $\triangleleft_{L}=<_{V_{1}}^{h}$.

Soit $\sigma$ un cône de $\mathcal{E}_{V}$ de dimension 2 (maximale) et $\left\{L_{1}, L_{2}\right\}=\mathcal{L}(\sigma)$ avec $L_{1}<L_{2}$. Notons $Q_{1}, \ldots, Q_{r}$ la base standard de $h(I)$ associée à $\sigma$. On définit $\kappa_{\sigma}^{1} \in \mathbb{N}$ par

$$
\kappa_{\sigma}^{1}=\max \left\{\operatorname{ord}^{V_{1}}\left(Q_{j}\right)-\operatorname{ord}^{V_{1}}\left(\sigma^{L_{2}}\left(Q_{j}\right)\right), j=1, \ldots, r\right\} .
$$

Avec les notations précédentes, nous avons (voir la figure 3 )

$$
\operatorname{ord}^{V_{1}}\left(\sigma^{L_{2}}\left(Q_{j}\right)\right)=\operatorname{ord}^{V_{1}}\left(\exp _{\triangleleft_{L_{2}}}\left(Q_{j}\right)\right) \text {. }
$$

Maintenant on définit $\kappa^{1} \in \mathbb{N}$ comme le maximum des $\kappa_{\sigma}^{1}$ pour les cônes $\sigma \in \mathcal{E}_{V}$ de dimension 2 . Voici une reformulation plus précise du théorème 2 . 


\section{R. BAHLOUL}

Théorème 2 BIS. Pour tout $w \in \mathbb{Z}^{2}$,

$$
\bar{V}_{w}(M) \subset V_{w+\left(\kappa^{1}, 0\right)}(M) .
$$

\subsection{Contrôle de la montée de l'ordre par rapport $V_{\mathbf{1}}$}

Soit $\sigma \in \mathcal{E}_{V}$ un cône de dimension maximale et soient $L_{1}<L_{2}$ ses générateurs primitifs. Soit $m \in \bar{V}_{w}(M)$ avec $w$ dans $\mathbb{Z}^{2}$, en particulier $m \in\left(V_{L_{1}(w)}^{L_{1}}\left(\mathcal{D}_{n+2}\right) \delta\right) \cap\left(V_{L_{2}(w)}^{L_{2}}\left(\mathcal{D}_{n+2}\right) \delta\right)$. Supposons donné $P \in \mathcal{D}_{n}$ tel que $P \delta=m$ et $\operatorname{ord}^{L_{1}}(P) \leqslant L_{1}(w)$ et tel que $\operatorname{ord}^{L_{2}}(P)>L_{2}(w)$. Alors nous avons montré dans le lemme 2.2 comment construire, en un nombre fini d'étapes, un élément $P_{\sigma}$ tel que $\operatorname{ord}^{L_{1}}\left(P_{\sigma}\right) \leqslant \operatorname{ord}^{L_{1}}(P)$ (i.e. l'ordre par rapport à $L_{1}$ n'a pas augmenté) et $\operatorname{ord}^{L_{2}}\left(P_{\sigma}\right) \leqslant L_{2}(w)$ (i.e. l'ordre par rapport à $L_{2}$ a baissé le plus possible). Nous pouvons nous demander ce qui se passe pour l'ordre $V_{1}$ de $P_{\sigma}$ par rapport à celui de $P$. Nous allons montrer que cet ordre peut augmenter mais de manière contrôlée. C'est l'objet du lemme suivant.

Lemme 3.1. Soit $\sigma$ un cône de dimension maximale de $\mathcal{E}_{V}(h(I))$ et $L_{1} \neq L_{2}$ ses générateurs primitifs (il est possible que $L_{i} \notin \sigma$ ). Supposons $V_{1} \leqslant L_{1}<L_{2} \leqslant V_{2}$.

Soient $w \in \mathbb{Z}^{2}$ et $m \in V_{L_{2}(w)}^{L_{2}}(M)$. Soit $P \in \mathcal{D}_{n+2}$ tel que $P \delta=m$ et $\operatorname{ord}^{L_{1}}(P) \leqslant L_{1}(w)$ alors on peut construire $P_{\sigma} \in \mathcal{D}_{n+2}$ à partir de $P$ tel que

i) $P_{\sigma}-P \in I$,

ii) $P_{\sigma} \in{ }^{\sigma} V_{w}\left(\mathcal{D}_{n+2}\right)$, en particulier ord ${ }^{L_{2}}\left(P_{\sigma}\right) \leqslant L_{2}(w)$,

iii) $\operatorname{ord}^{V_{1}}\left(P_{\sigma}\right) \leqslant \max \left\{\operatorname{ord}^{V_{1}}(P), w_{1}+\kappa_{\sigma}^{1}\right\}$.

C'est iii qui justifie l'intitulé de ce paragraphe.

Démonstration. Si ord ${ }^{L_{2}}(P) \leqslant L_{2}(w)$, il suffit de poser $P_{\sigma}=P$. On suppose donc que ord ${ }^{L_{2}}(P)>$ $L_{2}(w)$ ce qui entraine $\operatorname{ord}^{V_{1}}\left(\sigma^{L_{2}}(P)\right) \leqslant w_{1}$.

Par hypothèse il existe $P_{2} \in \mathcal{D}_{n+2}$ vérifiant $P_{2} \delta=m$ et $\operatorname{ord}^{L_{2}}\left(P_{2}\right) \leqslant L_{2}(w)$. On définit $H_{0}=$ $z^{l_{0}} h\left(P-P_{2}\right)=z^{l} h(P)-z^{l_{2}} h\left(P_{2}\right)$ (il existe des entiers $l_{0}, l$, et $l_{2}$ satisfaisant une telle égalité), $H=z^{l} h(P)$ et $H_{2}=z^{l_{2}} h\left(P_{2}\right)$. On reprend le début de la preuve du lemme 2.2 à la différence qu'on travaille avec la forme $L_{2}$ au lieu de $L_{1}$. On considère donc la division de $H_{0}$ par la base standard $Q_{1}, \ldots, Q_{r}$ relativement à l'ordre $\triangleleft_{L_{2}}$ ce qui donne :

$$
H_{0}=\sum_{j=1}^{r} q_{j} Q_{j} \quad \text { avec } \operatorname{ord}^{L_{2}}\left(H_{0}\right) \geqslant \operatorname{ord}^{L_{2}}\left(q_{j} Q_{j}\right) .
$$

On note $J$ l'ensemble des $j$ dans $\{1, \ldots, r\}$ pour lesquels l'inégalité précédente est une égalité. On pose alors

$$
W=\sum_{j \in J} \sigma^{L_{2}}\left(q_{j}\right) Q_{j} \text { et } H^{\prime}=H-W .
$$

Maintenant, ce qui nous intéresse, c'est la différence entre $\operatorname{ord}^{V_{1}}(H)$ et $\operatorname{ord}^{V_{1}}\left(H^{\prime}\right)$. C'est l'objet de ce qui suit.

Affirmations. Nous avons

a) $\operatorname{ord}^{V_{1}}(W) \leqslant w_{1}+\kappa_{\sigma}^{1}$,

b) $\operatorname{ord}^{V_{1}}(W)-\operatorname{ord}^{V_{1}}\left(\sigma^{L_{2}}(W)\right) \leqslant \kappa_{\sigma}^{1}$.

Démontrons ces affirmations. a) Nous avons ord ${ }^{V_{1}}\left(\sigma^{L_{2}}(W)\right)=\operatorname{ord}^{V_{1}}\left(\sigma^{L_{2}}(H)\right)=\operatorname{ord}^{V_{1}}\left(\sigma^{L_{2}}(P)\right)$ et ce dernier est majoré par $w_{1}$ donc si (b) est vrai il en est de même pour (a). 


\section{Existence DE POLYNÔMes DE Bernstein-SATO}

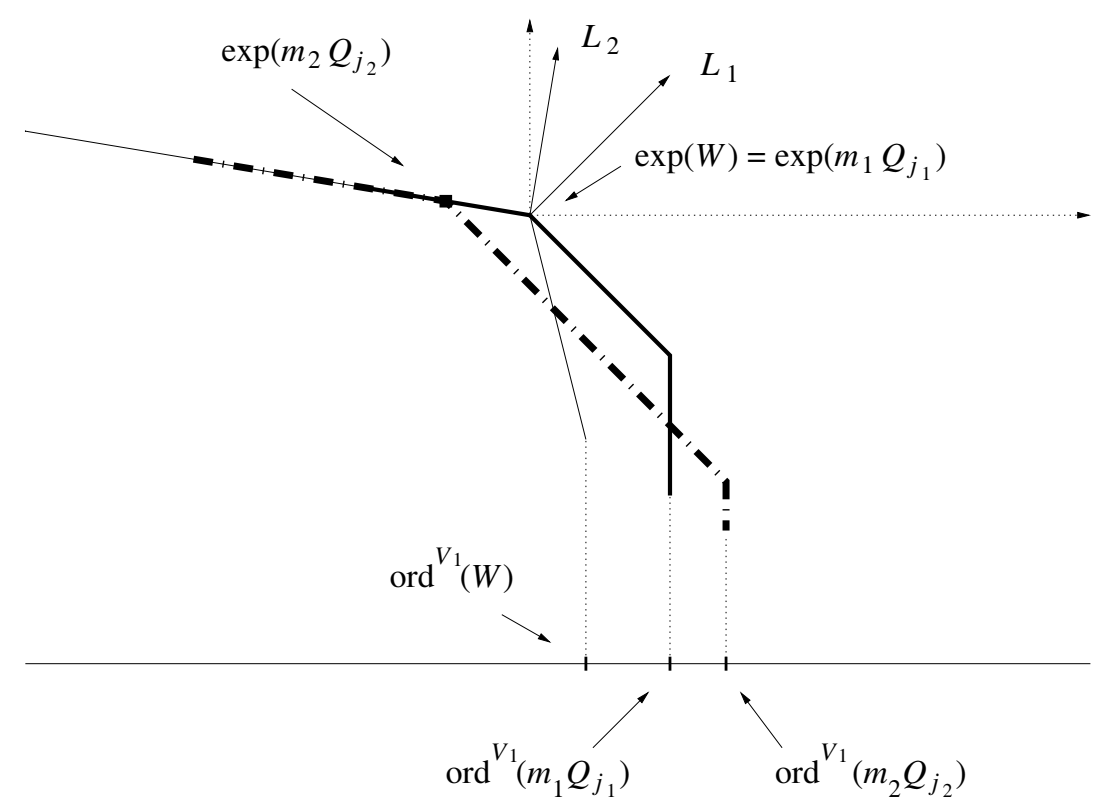

FiguRE 4. Illustration des affirmations.

b) Comme au paragraphe précédent, on peut montrer que la division de $W$ par $\left\{Q_{j}, j \in J\right\}$ relativement à $\triangleleft_{L_{2}}$ donne $W=\sum_{j \in J} \sigma^{L_{2}}\left(q_{j}\right) Q_{j}$. Par conséquent, il existe $j_{1} \in J$ tel que $\exp _{\triangleleft_{L_{2}}}(W)=$ $\exp _{\triangleleft_{L_{2}}}\left(m_{1} Q_{j_{1}}\right)$ où $m_{1}=\operatorname{mp}_{\triangleleft_{L_{2}}}\left(q_{j_{1}}\right)$ qui est un monône de $\sigma^{V_{1}}\left(\sigma^{L_{2}}\left(q_{j_{1}}\right)\right)$. En particulier, ceci implique

$$
\operatorname{ord}^{V_{1}}\left(\sigma^{L_{2}}(W)\right)=\operatorname{ord}^{V_{1}}\left(m_{1} \sigma^{L_{2}}\left(Q_{j_{1}}\right)\right)
$$

D'autre part,

$$
\operatorname{ord}^{V_{1}}(W) \leqslant \max \left\{\operatorname{ord}^{V_{1}}\left(\sigma^{L_{2}}\left(q_{j}\right) Q_{j}\right) ; j \in J\right\} .
$$

Soit alors $j_{2} \in J$ tel que ord ${ }^{V_{1}}\left(\sigma^{L_{2}}\left(q_{j_{2}}\right) Q_{j_{2}}\right)=\max \left\{\operatorname{ord}^{V_{1}}\left(\sigma^{L_{2}}\left(q_{j}\right) Q_{j}\right), j \in J\right\}$. En prenant $m_{2}=$ $\operatorname{mp}_{\triangleleft_{L_{2}}}\left(q_{j_{2}}\right)$, on obtient

$$
\operatorname{ord}^{V_{1}}(W) \leqslant \operatorname{ord}^{V_{1}}\left(m_{2} Q_{j_{2}}\right) .
$$

Remarquons qu'il est possible qu'on ait $j_{1}=j_{2}$. Cependant, on a toujours l'affirmation suivante (voir la figure 4).

Affirmation. Nous avons

c) $\operatorname{ord}^{V_{1}}\left(m_{2} \sigma^{L_{2}}\left(Q_{j_{2}}\right)\right) \leqslant \operatorname{ord}^{V_{1}}\left(m_{1} \sigma^{L_{2}}\left(Q_{j_{1}}\right)\right)$.

En utilisant cette affirmation et les identités (5) et (6), nous obtenons

$$
\begin{aligned}
\operatorname{ord}^{V_{1}}(W)= & \operatorname{ord}^{V_{1}}(W)-\operatorname{ord}^{V_{1}}\left(m_{2} Q_{j_{2}}\right)+\operatorname{ord}^{V_{1}}\left(m_{2} Q_{j_{2}}\right) \\
& -\operatorname{ord}^{V_{1}}\left(m_{2} \sigma^{L_{2}}\left(Q_{j_{2}}\right)\right)+\operatorname{ord}^{V_{1}}\left(m_{2} \sigma^{L_{2}}\left(Q_{j_{2}}\right)\right) \\
& -\operatorname{ord}^{V_{1}}\left(m_{1} \sigma^{L_{2}}\left(Q_{j_{1}}\right)\right)+\operatorname{ord}^{V_{1}}\left(\sigma^{L_{2}}(W)\right) \\
\leqslant & \operatorname{ord}^{V_{1}}\left(m_{2} Q_{j_{2}}\right)-\operatorname{ord}^{V_{1}}\left(m_{2} \sigma^{L_{2}}\left(Q_{j_{2}}\right)\right)+\operatorname{ord}^{V_{1}}\left(\sigma^{L_{2}}(W)\right) \\
\leqslant & \kappa_{\sigma}^{1}+\operatorname{ord}^{V_{1}}\left(\sigma^{L_{2}}(W)\right) .
\end{aligned}
$$

Ceci démontre le point (b). Il ne reste plus qu'à démontrer le point (c). 


\section{R. BAHLOUL}

La division de $\sigma^{L_{2}}(W)$ par $\left\{\sigma^{L_{2}}\left(Q_{j}\right), j \in J\right\}$ relativement à $\triangleleft_{L_{2}}$ donne le résultat suivant :

$$
\sigma^{L_{2}}(W)=\sum_{j \in J} \sigma^{L_{2}}\left(q_{j}\right) \sigma^{L_{2}}\left(Q_{j}\right)
$$

Par conséquent,

Or

$$
\exp _{\triangleleft_{L_{2}}}\left(\sigma^{L_{2}}\left(q_{j_{2}}\right) \sigma^{L_{2}}\left(Q_{j_{2}}\right)\right) \unlhd_{L_{2}} \exp _{\triangleleft_{L_{2}}}\left(\sigma^{L_{2}}(W)\right) .
$$

et

$$
\exp _{\triangleleft_{L_{2}}}\left(\sigma^{L_{2}}(W)\right)=\exp _{\triangleleft_{L_{2}}}(W)=\exp _{\triangleleft_{L_{2}}}\left(m_{1} \sigma^{L_{2}}\left(Q_{j_{1}}\right)\right)
$$

$$
\exp _{\triangleleft_{L_{2}}}\left(\sigma^{L_{2}}\left(q_{j_{2}}\right) \sigma^{L_{2}}\left(Q_{j_{2}}\right)\right)=\exp _{\triangleleft_{L_{2}}}\left(m_{2} \sigma^{L_{2}}\left(Q_{j_{2}}\right)\right)
$$

donc

$$
\exp _{\triangleleft_{L_{2}}}\left(m_{2} \sigma^{L_{2}}\left(Q_{j_{2}}\right)\right) \unlhd_{L_{2}} \exp _{\triangleleft_{L_{2}}}\left(m_{1} \sigma^{L_{2}}\left(Q_{j_{1}}\right)\right) .
$$

Or $\operatorname{ord}^{L_{2}}\left(m_{2} \sigma^{L_{2}}\left(Q_{j_{2}}\right)\right)=\operatorname{ord}^{L_{2}}\left(m_{1} \sigma^{L_{2}}\left(Q_{j_{1}}\right)\right)$, donc

$$
\operatorname{ord}^{V_{1}}\left(m_{2} \sigma^{L_{2}}\left(Q_{j_{2}}\right)\right) \leqslant \operatorname{ord}^{V_{1}}\left(m_{1} \sigma^{L_{2}}\left(Q_{j_{1}}\right)\right) .
$$

Le point (c) est démontré.

Voyons maintenant comment l'affirmation (a) permet de montrer le troisième point du lemme. Nous sommes partis de $H$ et nous avons construit $H^{\prime}=H-W$. Par (a), nous avons ord ${ }^{V_{1}}\left(H^{\prime}\right) \leqslant$ $\max \left(\operatorname{ord}^{V_{1}}(H), w_{1}+\kappa_{\sigma}^{1}\right)$. La suite consiste à faire les mêmes opérations avec $H^{\prime}$ à la place de $H$. Le dernier élément $H_{\sigma}$ ainsi construit vérifie les relations $H_{\sigma}-H \in h(I)$ et $\operatorname{ord}^{V_{1}}\left(H_{\sigma}\right) \leqslant$ $\max \left(\operatorname{ord}^{V_{1}}(H), w_{1}+\kappa_{\sigma}^{1}\right)$. On pose alors $P_{\sigma}=H_{\sigma \mid z=1}$, on a bien $P_{\sigma}-P \in I$ et $\operatorname{ord}^{V_{1}}\left(P_{\sigma}\right) \leqslant$ $\max \left(\operatorname{ord}^{V_{1}}(P), w_{1}+\kappa_{\sigma}^{1}\right)$. Le lemme est démontré.

\subsection{Fin de la preuve}

Démonstration du théorème 2 bis. Notons $L_{0}=V_{1}<\cdots<L_{q}=V_{2}$ les éléments primitifs du 1squelette de $\mathcal{E}_{V}$. Pour chaque $i=1, \ldots, q$, notons $\sigma_{i} \in \mathcal{E}_{V}$ le cône contenant le cône ouvert engendré par $L_{i-1}$ et $L_{i}$.

Soit $m \in \bar{V}_{w}(M)$. Montrons par récurrence sur $i$ que pour tout $i=0, \ldots, q$, il existe $T_{i} \in \mathcal{D}_{n+2}$ vérifiant

i) $T_{i} \delta=m$,

ii) $T_{i} \in V_{L_{i}(w)}^{L_{i}}(M)$,

iii) $\operatorname{ord}^{V_{1}}\left(T_{i}\right) \leqslant w_{1}+\kappa^{1}$.

Pour $i=0, m \in \bar{V}_{w}$; donc en particulier $m \in V_{V_{1}(w)}^{V_{1}}$ (notons que $V_{1}(w)=w_{1}$ ), donc il existe $T_{0}$ tel que $T_{0} \delta=m$ et $\operatorname{ord}^{V_{1}}\left(T_{0}\right) \leqslant w_{1} \leqslant w_{1}+\kappa^{1}$.

Supposons l'assertion vraie au rang $i-1$. On applique le lemme 3.1 avec $\sigma=\sigma_{i}$ et $P=T_{i-1}$. On pose alors $T_{i}=P_{\sigma}$ (notations du lemme).

D'après ce même lemme, $T_{i}$ vérifie $\mathrm{i}$, ii et $\operatorname{ord}^{V_{1}}\left(T_{i}\right) \leqslant \max \left(\operatorname{ord}^{V_{1}}\left(T_{i-1}\right), w_{1}+\kappa^{1}\right)=w_{1}+\kappa^{1}$, c'est-à-dire l'inégalité iii.

Ainsi, l'assertion est vraie pour tout $i$. En particulier, pour $i=q$, on a $m=T_{q} \delta$, ord ${ }^{V_{2}}\left(T_{q}\right) \leqslant w_{2}$ et $\operatorname{ord}^{V_{1}}\left(T_{q}\right) \leqslant w_{1}+\kappa^{1}$, c'est-à-dire $m \in V_{w+\left(\kappa^{1}, 0\right)}(M)$.

Remarque 3.2. Le procédé de construction de $\left(\kappa^{1}, 0\right)$ montre qu'un tel $\kappa$ n'est à priori pas unique. En effet, en inversant les rôles de $V_{1}$ et $V_{2}$, on aurait pu construire un $\kappa$ de la forme $\left(0, \kappa^{2}\right)$. 


\section{Existence De POLynômes De Bernstein-Sato}

\section{REMERCIEMENTS}

Je remercie mon directeur de thèse Michel Granger pour les conseils donnés durant l'élaboration de ce travail qui constitue une partie de ma thèse préparée à l'université d'Angers. Je remercie le rapporteur anonyme pour ses questions et remarques qui ont permis notamment d'améliorer la démonstration de la proposition 2.1.

\section{BIBLIOGRAPHIE}

ACG01 A. Assi, F. J. Castro Jiménez et M. Granger, The analytic standard fan of a $\mathcal{D}$-module, J. Pure Appl. Algebra 164 (2001), 3-21.

Bah03 R. Bahloul, Contributions à l'étude des idéaux de Bernstein-Sato d'un point de vue constructif, Thèse de Doctorat, Université d'Angers (2003).

Ber72 I. N. Bernstein, The analytic continuation of generalised functions with respect to a parameter, Funct. Anal. Appl. 6 (1972), 273-285.

Bjö73 J. E. Björk, Dimensions over algebras of differential operators, Preprint (1973).

CN97 F. J. Castro Jiménez et L. Nárvaez-Macarro, Homogenising differential operators, Preprint (1997).

Gyo93 A. Gyoja, Bernstein-Sato's polynomial for several analytic functions, J. Math. Kyoto Univ. 33 (1993), 399-411.

Kas76 M. Kashiwara, B-functions and holonomic systems. Rationality of roots of B-functions, Invent. Math. 38 (1976), 33-53.

Lic88 B. Lichtin, Generalized Dirichlet series and b-functions, Compositio Math. 65 (1988), 81-120.

Mal75 B. Malgrange, Le polynôme de Bernstein d'une singularité isolée, Lecture Notes in Mathematics, vol. 459 (Springer, Berlin, 1975), 98-119.

Sab87a C. Sabbah, Proximité évanescente I. La structure polaire d'un D-module, Appendice en collaboration avec F. J. Castro Jiménez, Compositio Math. 62 (1987), 283-328.

Sab87b C. Sabbah, Proximité évanescente II. Équations fonctionnelles pour plusieurs fonctions analytiques, Compositio Math. 64 (1987), 213-241.

Rouchdi Bahloul rouchdi@math.kobe-u.ac.jp

Department of Mathematics, Faculty of Science, Kobe University, 1-1 Rokkodai, Nada-ku, Kobe 657-8501, Japan 